EPJ Web of Conferences 114,02032 (2016)

DOI: 10.1051/epjconf/201611402032

(C) Owned by the authors, published by EDP Sciences, 2016

\title{
Turbulent transport measurements in a cold model of GT-burner at realistic flow rates
}

\author{
Oleg Gobyzov ${ }^{1,2}$, Leonid Chikishev ${ }^{1,2}$, Alexey Lobasov ${ }^{1,2}$, Dmitriy Sharaborin ${ }^{1,2}$, Vladimir Dulin ${ }^{1,2}$, Artur Bilsky ${ }^{1,2}$, \\ Vakhtang Tsatiashvili ${ }^{3}$, Valery Avgustinovich ${ }^{3}$ and Dmitriy Markovich ${ }^{1,2}$
}

\author{
${ }^{1} K u t a t e l a d z e$ Institute of Thermophysics, Siberian Branch of Russian Academy of Sciences, 630090, Novosibirsk, Russia \\ ${ }^{2}$ Novosibirsk State University, 630090, Novosibirsk, Russia \\ ${ }^{3}$ JSC Aviadvigatel 614010, Perm, Russia
}

\begin{abstract}
In the present work simultaneous velocity field and passive admixture concentration field measurements at realistic flow-rates conditions in a non-reacting flow in a model of combustion chamber with an industrial mixing device are reported. In the experiments for safety reasons the real fuel (natural gas) was replaced with neon gas to simulate stratification in a strongly swirling flow. Measurements were performed by means of planar laser-induced fluorescence (PLIF) and particle image velocimetry technique (PIV) at Reynolds number, based on the mean flow rate and nozzle diameter, $\approx 300000$. Details on experimental technique, features of the experimental setup, images and data preprocessing procedures and results of performed measurements are given in the paper. In addition to the raw velocity and admixture concentration data in-depth evaluation approaches aimed for estimation of turbulent kinetic energy (TKE) components, assessment of turbulent Schmidt number and analysis of the gradient closure hypothesis from experimental data are presented in the paper.
\end{abstract}

\section{Introduction}

Numerical codes for turbulent mixing calculations for GT burners require verification by comprehensive experimental data, which is often a challenging task to provide. Conventional single-point measurements usually have high temporal resolution but do not provide information about spatial features of the flow and turbulent transport. Contrary to that, planar optical methods, like PIV and PLIF can take advantage of capturing the instantaneous distributions of flow velocity and passive admixture concentration. Such data are usually more adequate for numerical code verification.

Fuel-air mixing plays one of key roles in flame stabilization and pollutant formation. Computational fluid dynamics code validation, using for complex swirling industrial flows demands on reliable experimental data. In particular, one of the key parameters for computational model verification is turbulent transport of passive admixture. Laser-induced fluorescence of acetone vapor (acetone PLIF) in combination with particle image velocimetry (PIV) can be used for quantitative measurements of local fuel/air ratio at constant temperature and pressure without chemical reaction [1]. There are several advantages of the acetone as a tracer in comparison with other polyatomic molecules: a high vapor pressure at room temperature, an absorption feature accessible with high-pulse-energy lasers, short emission

\footnotetext{
a Corresponding author: oleg.a.g.post@gmail.com
}

lifetime, low toxicity, low cost for seeding at high flowrates, compatibility with air, insensitivity to effects of collisional quenching, and high fluorescence signal levels. At room temperature and atmospheric pressure acetone had a broadband absorption feature which extends from 225 to $320 \mathrm{~nm}$ with a peak near $275 \mathrm{~nm}$. Fluorescence in the wavelength range between 350 and $550 \mathrm{~nm}$ is emitted from the first excited singlet state $\mathrm{S} 1$, following laser excitation from the ground electronic state S0. For example authors of paper [2] investigated turbulent mass-transport for transverse jet flows of laboratory scale.

Full cycle of PIV/PLIF experiment is not limited to the retrieving of raw experimental data, which can undergo certain distortions due to experimental conditions or limitations of the measurement technique. Such data requires proper evaluation to provide more complex and reliable basis for analysis and validation of CFD codes. Present paper is focused on flow structure measurements and mass-transfer investigation in premixing GT-burner. Details of the experimental techniques, description of the GT burner model apparatus, as well as methods of assessment and evaluation of the raw low-repetition experimental data are given. 


\section{Experimental setup and conditions}

The measurements were carried out in cold combustion chamber model (see Fig. 1). The burner was provided by JSC Aviadvigatel (Perm, Russia). The burner with diameter $d=80 \mathrm{~mm}$ has two channels for fuel supply, but in the present study fuel was supplied only through the central channel. Main air flow was supplied from compressed air line into the plenum chamber through four hoses (intake collector). Turbulence generating grid was installed. Inside the plenum chamber and the coaxial swirler of the burner generated high-swirl premixed flow. The model fuel (neon gas) was supplied to the central channel of the burner. Test section of combustion chamber was made of quartz cylinder $(120 \mathrm{~mm}$ inner diameter). An optical section was $170 \mathrm{~mm}$ long.

The maximum air flow-rate was up to $0.5 \mathrm{~kg} / \mathrm{s}$, the temperature of the air was $17{ }^{\circ} \mathrm{C}$. The Reynolds number based on the nozzle diameter, and the bulk velocity was about $3 \cdot 10^{5}$. The pressure inside the chamber was $2 \mathrm{~atm}$. with pressure drop of $4 \%$ at the premixer. To model stratification in swirling flow and to keep experiment safe at the same time neon gas was used instead of methane. The gas was supplied from a $10 \mathrm{~atm}$. Pressure tank. The neon flowrate was controlled using Bronkhorst flowmeters. Water-glycerol particles were used to provide PIV measurements (the main air flow was seeded). The seeder was connected to the main air pipe via bypass with 0.5 atm pressure difference between inlet and exit of the seeder. The temperature stabilized acetone bubbler was employed to seed the neon flow for PLIF measurements. Neon massflow rate was $5.4 \mathrm{~g} / \mathrm{s}$. Owing to temperature stabilization at constant flow-rate the acetone vapor concentration in neon flow also remained constant.

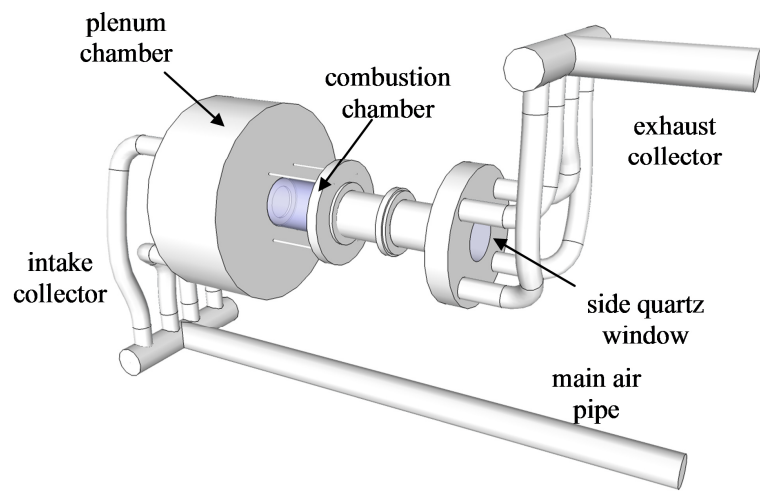

Figure 1. 3D sketch of the experimental setup.

An arrangement of the setup for combined PIV/PLIF measurements is depicted in Fig. 2.An orientation of coordinate axis is also shown in the fig. 2 and coordinates will be referred through the paper according to the figure. PIV system included double pulsed Nd:YAG laser (Quantel Ever Green 200) and 4M CCD camera ImperX IGV-B2020 supplied with the lens SIGMA AF $50 \mathrm{~mm}$ (F2,8 EX DG Macro) optical band-pass filter (Edmund Optics, $532 \pm 10 \mathrm{~nm}, 60 \%$ transmittance). To deliver the laser sheet inside the test section and to minimize scattering and reflections of the beam side quartz window (see fig. 1) was installed at the end of the cylindrical section. PLIF system included Quantel Brilliant B Nd:YAG laser and ICCD camera PI-MAX-4 (Princeton Instruments). Quantum efficiency of the s20 photocathode in spectral region $290-320 \mathrm{~nm}$ is about 25 $\%$. LaVision UV-lens (f\#2.8, $100 \mathrm{~mm})$ with interference band-pass optical filter $(280-600 \mathrm{~nm})$ and multi-notch filter $(1064,532,355 \mathrm{~nm})$ was installed on the camera. Exposure time of ICCD camera in experiments was 200 ns. Fourth harmonic of Nd:YAG laser $(266 \mathrm{~nm})$ was used to excite fluorescence of the acetone vapor. Average pulse energy measured with Coherent LabMax Top was $70 \mathrm{~mJ}$. To provide quantitative measurements collimated Sheet Optics (LaVision) was used. These two systems were synchronized using pulse generator (Berkeley Nucleonics BNC 575 TTL). Green laser pulse to pulse delay was $2 \mu$ s to prevent particle loss due to out-of-plane motion. ICCD camera exposition was triggered between PIV laser pulses.

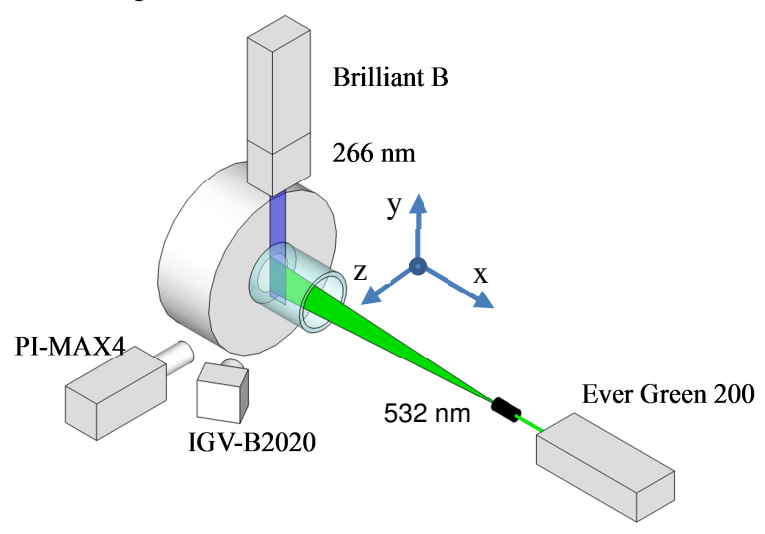

Figure 2. Scheme of the PIV/PLIF system arrangement.

A sheet of sensitive paper was placed into the measurement volume and two single shots of 266 and $532 \mathrm{~nm}$ lasers were done after each run to ensure that . PIV and PLIF measurement regions are well aligned. To measure magnification factor and the mapping functions of two cameras a calibration target was placed inside the cylinder.. In several minutes after seeding start the waterglycerol particles formed a film on a chamber wall making the cylinder non-transparent. Thus maximum statistics per one run was about 300 couples of images at $5 \mathrm{~Hz}$ frame-rate. The full statistics of 4000 fields of velocity and concentration respectively was collected in several consecutive experiments.

Besides the simultaneous PIV/PLIF experiments separate PIV experiments were performed in YZ plane at the distance of $0.1 \cdot d$ and $1 \cdot d$ from the mixing device exit to ensure that the flow is axisymmetric. 


\section{Images and data processing}

\subsection{PIV Image processing}

Due to inclusions in side surface of the cylinder laser sheet used for PIV also had large number of stripy inhomogeneities. It resulted in distorted data retrieved from PIV evaluation: stripy structure emerged in average velocity fields, and especially in higher order momentums. An approach similar to light sheet correction in PLIF was adapted to correct an intensity of particle images. Since the inhomogeneities were produced by stationary defects in the cylinder material, intensity of each pixel was corrected by the timeaveraged intensity:

$$
I^{*}(X, Y)=\frac{I(X, Y)}{\langle I(X, Y)\rangle} I_{0}
$$

where $\mathrm{I}_{0}$ is the mean of the time-averaged intensity:

$$
I_{0}=\frac{1}{n} \sum_{x} \sum_{y}\langle I(X, Y)\rangle
$$

This procedure, although not fully compensating lighting flaws, substantially improved quality of particle images and reduced impact of "stripes" on velocity fields.

Since in simultaneous PIV/PLIF measurements PIV camera was inclined to the measurement plane a correction of the perspective distortions was applied to the PIV images. For calibration of the camera second order polynomial model [3] was used.

Spatial resolution, as reported in $[4,5]$ and many other works can have a substantial impact on the results of investigation. Apparently, PIV measurements in highReynolds number flow usually unable to fetch turbulent features at small scales, but the higher spatial resolution is, the larger part of the turbulent spectra would be resolved. One should also take into account that PIV resolution is bounded above due to spatial averaging over the light sheet thickness. In the present experiments light sheet thickness $(\approx 1 \mathrm{~mm})$ didn't exceed the size of interrogation window.

There are two ways to vary a spatial resolution in PIV experiment: variation of optical magnification factor and the size of interrogation window in PIV algorithm. In the experiments under consideration it was important to perform measurements across the whole test section, and therefore optical magnification was selected to satisfy this condition and was fixed during the series of measurements. As for the variation of interrogation window, excessive reduction of its size can result in emergence of intense data noise. If higher order statistical moments or spatial derivatives are to be retrieved from PIV velocity fields, the noise amplification issue should be considered.

To assess an effect of spatial resolution $\Delta$, or rather of the interrogation window size, velocity field calculation was performed with final window size varied from $16 \times 16$ pix. to $128 \times 128$ pix. (see fig. 3). Even with qualitative analysis, it can be concluded that $16 \times 16$ pix. window interrogation introduces large amount of high-frequency noise, which contributes into the measured turbulence statistics, and if smoothed, distorts the profile in the area of low velocity pulsations. In its turn $128 \times 128$ pix. window interrogation results in substantial underestimation of spatial derivatives component, especially in the mixing layer. For $32 \times 32$ pix. and $64 \times 64$ pix. window almost no high-frequency noise component is observed, but larger interrogation window predictably yields a bit lower intensity of velocity pulsations. Same arguments can be applied to the second-order of velocity derivatives. In the final analysis PIV data were processed using final interrogation window size $\Delta$ of $32 \times 32$ pix. Peak validation and adaptive median filter $(5 \times 5)$ were employed to detect false vectors. In turbulent statistics false vectors were just excluded from evaluation, but for the calculation of spatial derivatives moving average interpolation of false vectors was applied to form a datasets on a regular grid. Since in all cases spatial overlap rate between the interrogation areas was $75 \%$, the grid step $h$ was four times smaller than $\Delta$. The final spatial resolution was 2 vectors per $1 \mathrm{~mm}$.

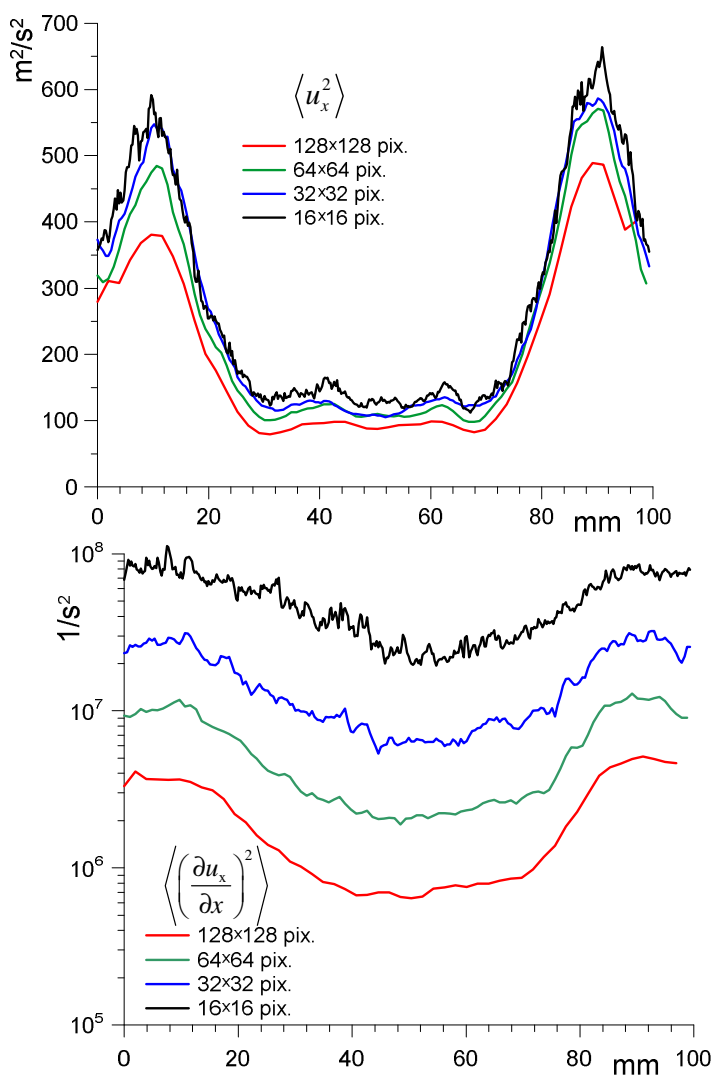

Figure 3 TKE component and second-order of velocity derivative profiles along $\mathrm{Y}$ axis at the distance of $22 \mathrm{~mm}$ from the mixing device exit.

Evaluation of more comprehensive descriptive parameters of turbulence and mixing processes, such as turbulent kinetic energy (TKE) dissipation rate and turbulent Schmidt number, both require calculation of velocity special derivatives. In its turn, numerical differentiation scheme for spatial derivatives calculation should be carefully chosen in order to keep proper balance between measurement noise amplification and truncation error. 
For the case under consideration several numerical differential schemes were considered for calculation of spatial velocity derivatives (see. Fig 4). Among these schemes were: simple central differentiation (eq. 3), least-squares (eq. 4) scheme with different point spacing $H$, proposed in [3], and Richardson extrapolation scheme optimized for minimization of random error amplification (eq.5).

$$
\begin{aligned}
& \frac{\partial u(x)}{\partial x}=\frac{u(x+H)-u(x-H)}{2 H} \\
& \frac{\partial u(x)}{\partial x}=\frac{2 u(x+2 H)+u(x+H)-u(x-H)-2 u(x-2 H)}{10 H} \\
& \frac{\partial u(x)}{\partial x}=\frac{16 u(x+4 H)+u(x+H)-u(x-H)-16 u(x-4 H)}{130 H}
\end{aligned}
$$

Profile of the second-order velocity derivative in a transversal cross-section of the measurement area at the distance of 1d from burner exit evaluated with different numerical derivation schemes is shown in fig.4. It can be seen, that simple central differentiation scheme and leastsquares with $H=h$ introduce amount off noise comparable to the signal amplitude, while for least-square scheme with $H \geq 2 h$ amount of high-frequency noise doesn't differ much, but truncation error increases significantly with increase of $H$. Note that least-square with $H=2 h$ and Richardson scheme with $H=1 h$ yield almost similar results because terms $u(x \pm 4 h)$ contribute most in both cases. According to this results, least-squares differential scheme with $H=2 h$ was chosen for further evaluation.

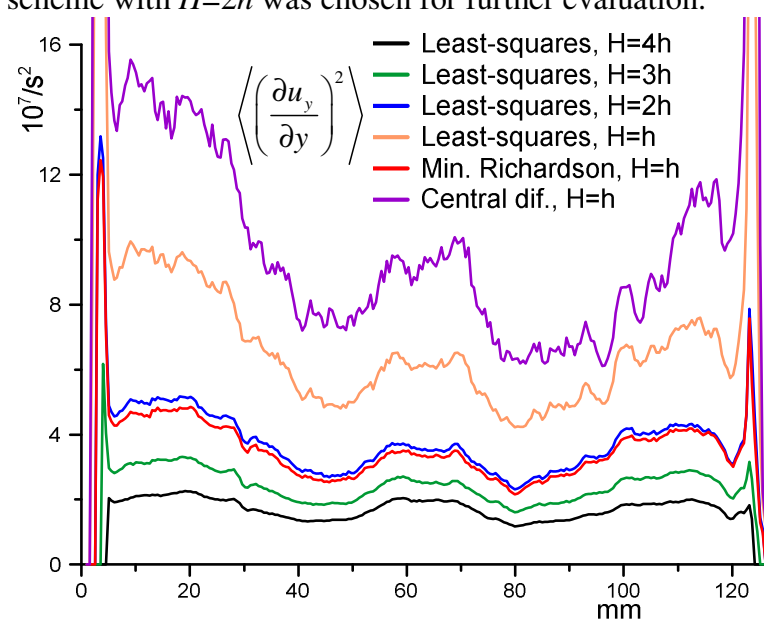

Figure 4 Second-order velocity derivative profile along $\mathrm{Z}$ axis at the distance of $1 \mathrm{~d}$ from the mixing device exit evaluated with different numerical derivation schemes

\subsection{PLIF Image processing}

As it is known, in PLIF the linear dependency of the fluorescence signal and the acetone tracer concentration are provided only if the energy flux in the light sheet is below the saturation level and absorption can be neglected. Linear response of acetone vapor was previously reported by Bryant et. al. at maximum energy flux of $353 \mathrm{~mJ} / \mathrm{cm}^{3}$ when excited at $266 \mathrm{~nm}$ (10 ns pulse width) [6]. The maximum laser energy flux in the experiment under consideration was more than two times lower $-140 \mathrm{~mJ} / \mathrm{cm}^{3}$. The linearity of the signal was verified prior to measurements and no evidence of saturation was found.

The raw PLIF images were processed using background subtraction and laser sheet correction procedures. After background subtraction images were divided by a reference sensitivity image. The reference sensitivity image was recorded while the test section was flooded with an acetone tracer at homogeneous concentration (averaged over 200 images). This procedure accounts for the angular lens light collection efficiency, variations of sensitivity of the camera, the quantum efficiency of fluorescence and spatial variations of laser intensity. In order to correct for additional shotto-shot fluctuations, each single image was corrected according to laser intensity deviation at the same time. Intensity variations were measured using energy meter. Fig. 5 shows an example of PLIF image evaluation (raw image and image after all correction procedures). The laser sheet direction is from top to bottom. The systematic error was significantly reduced using correction procedures.
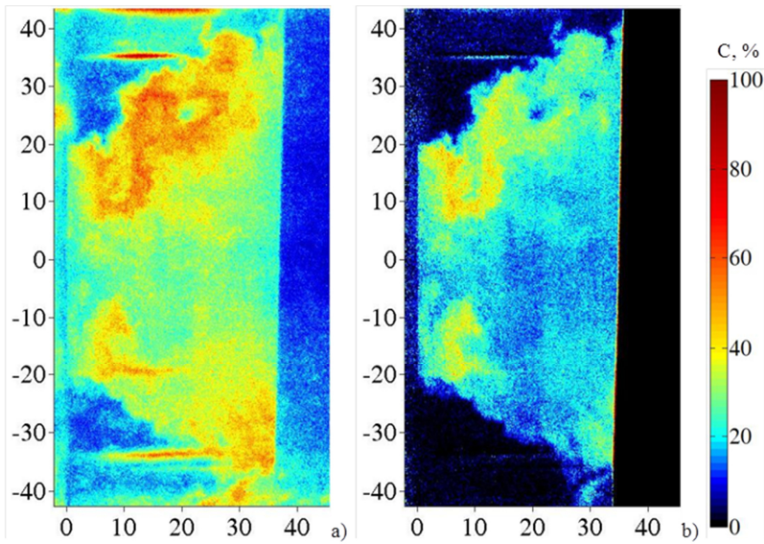

Figure 5 Instantaneous raw acetone PLIF image (a) and image after all correction procedures (b)

\section{Results and discussion}

\subsection{Velocity and admixture concentration distributions}

Separate PIV measurements taken in YZ have proved that in general flow has an axisymmetric structure, albeit some skewness, especially in radial component can be observed (see fig. 6). 
EFM 2015
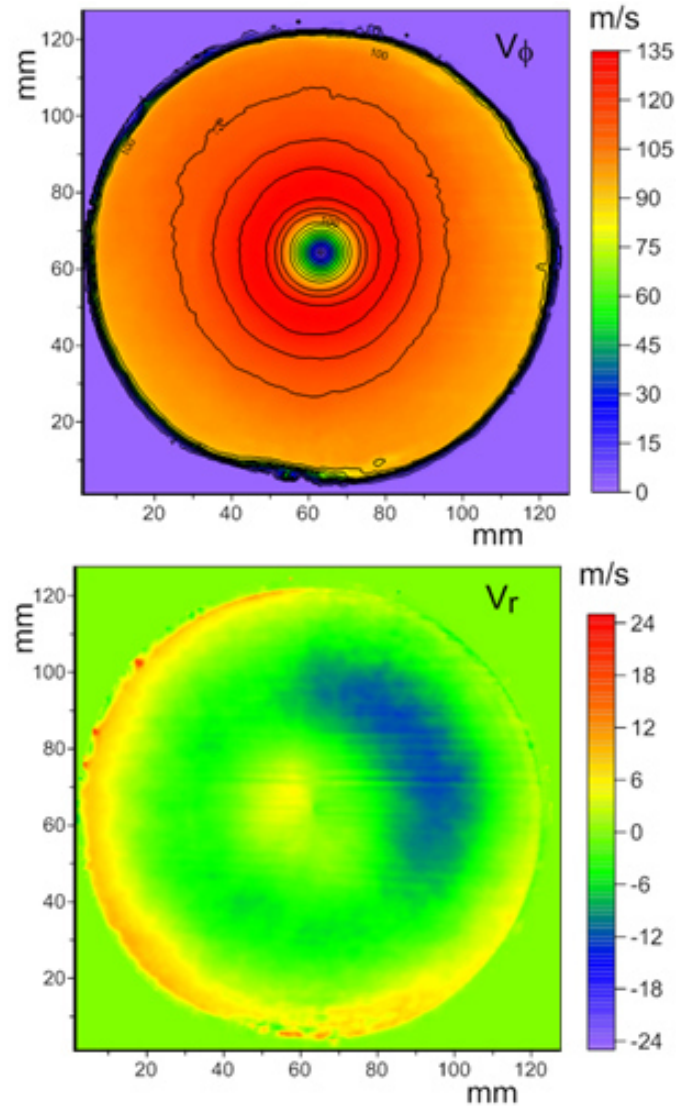

Figure 6. Average velocity components distribution in $\mathrm{YZ}$ plane at the distance of $1 \mathrm{~d}$ from mixing device exit.

Averaged velocity field in XY plane is presented in Fig. 7 (red line indicates the backflow region). As it is seen the flow is strongly swirled and after a sudden expansion backflow region appears. The maximum velocity at the burner exit is up to $80 \mathrm{~m} / \mathrm{s}$. Distinctive feature of the flow is a well pronounced recirculation region extending to the distance of one calibre. The central region of the flow exhibits jet flow due to model fuel supply at the center of the burner. The velocity maximum region reaches the wall at approximately $15 \mathrm{~mm}$ downstream of the burner. The corner recirculation region is not resolved due to poor signal/noise ratio at the chamber wall caused by laser reflections from the burner. More detailed quantitative description of the flow structure is available from the velocity profiles (fig. 8 and fig. 9). Axial and radial velocity components are presented for two distances downstream of the burner $(0,1 d$ and $0,5 d$ respectively).

The prominent features of the mean axial velocity profiles are the inflow region with high velocities and pronounced recirculation zone with mean velocities of the order $-10 \mathrm{~m} / \mathrm{s}$. The RMS values increases slightly with a distance from the axis of symmetry of the burner and have peaks in the inner and outer shear layers. Another feature of the flow is the central jet flow with axial velocity up to $10 \mathrm{~m} / \mathrm{s}$. Radial component of the velocity have its maxima only at the initial region of the flow. Downstream the burner mean radial velocity is approaches zero with RMS about $10 \mathrm{~m} / \mathrm{s}$.
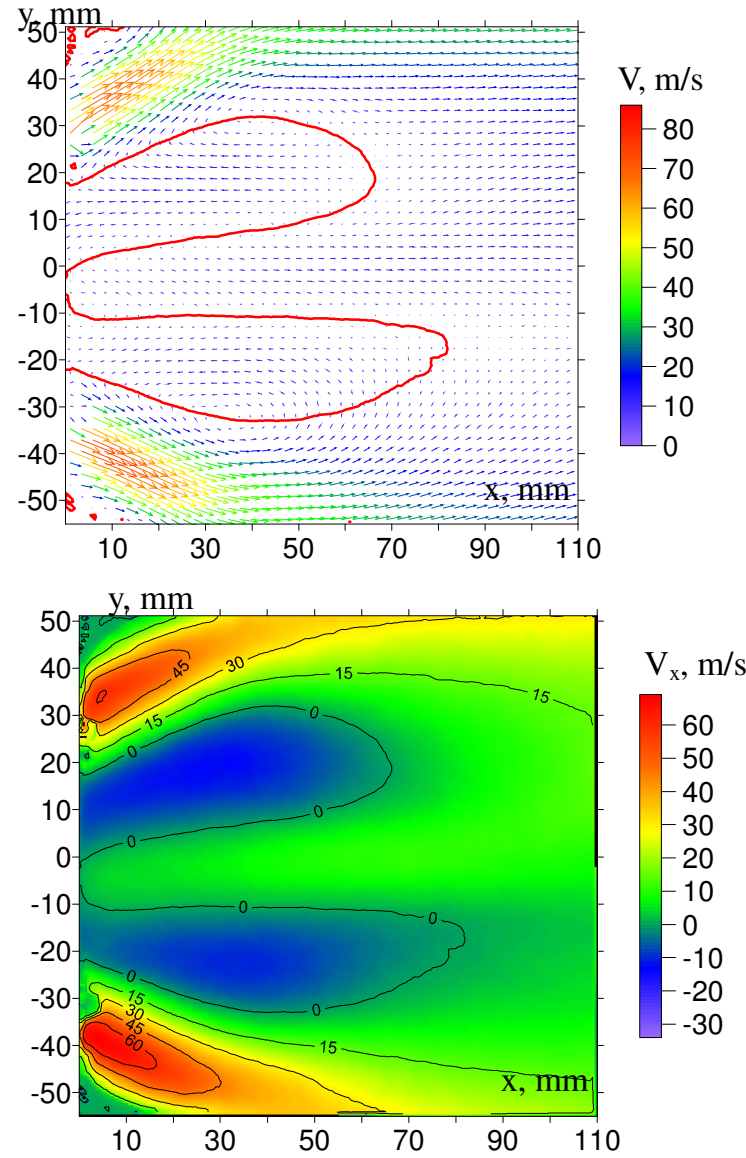

Figure 7. Averaged velocity field (upper) and axial velocity component field (lower).

As for the concentration distribution, it can be seen from figure 10 that downstream of the nozzle maximum mean concentration decreases 1.6 times. Another feature is that near the burner concentration profile has two wellpronounced maxima. This maxima indicates strong interaction of the central fuel jet with coaxial swirling flow. At the distance of $\approx 0.5 d$ from the burner only one central maxima of concentration is present. The concentration pulsations are quite high near the burner exit (about $30 \%$ of the maximum) and reaches its maximum in the inner shear layer of coaxial swirling flow. 

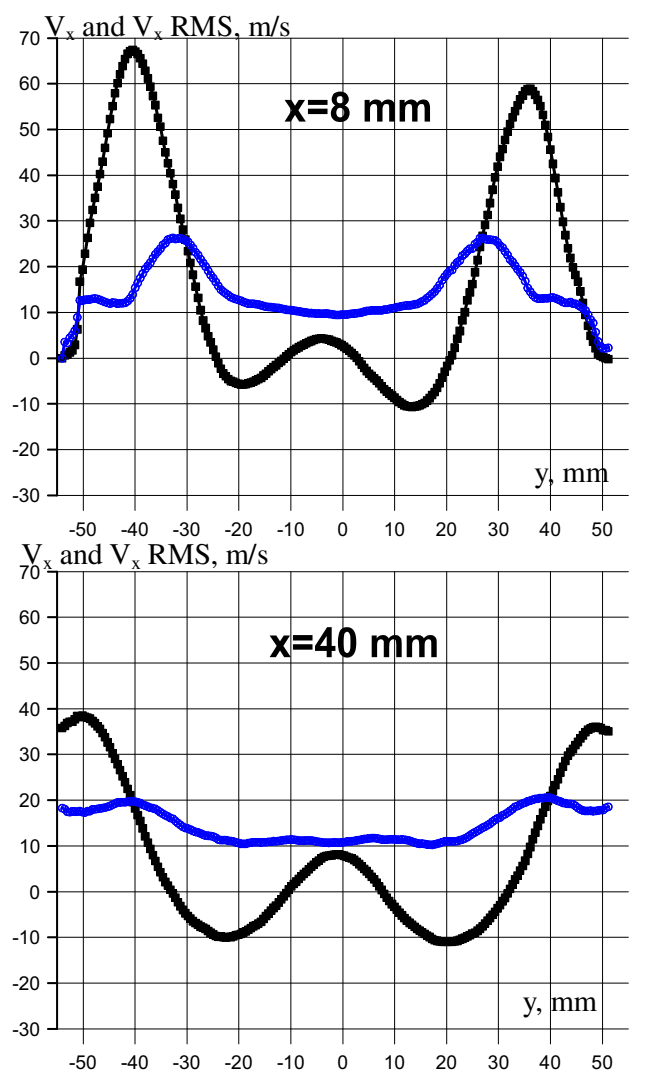

fig 8 Radial profiles of the mean (black) and RMS (blue) values of the axial $V x$
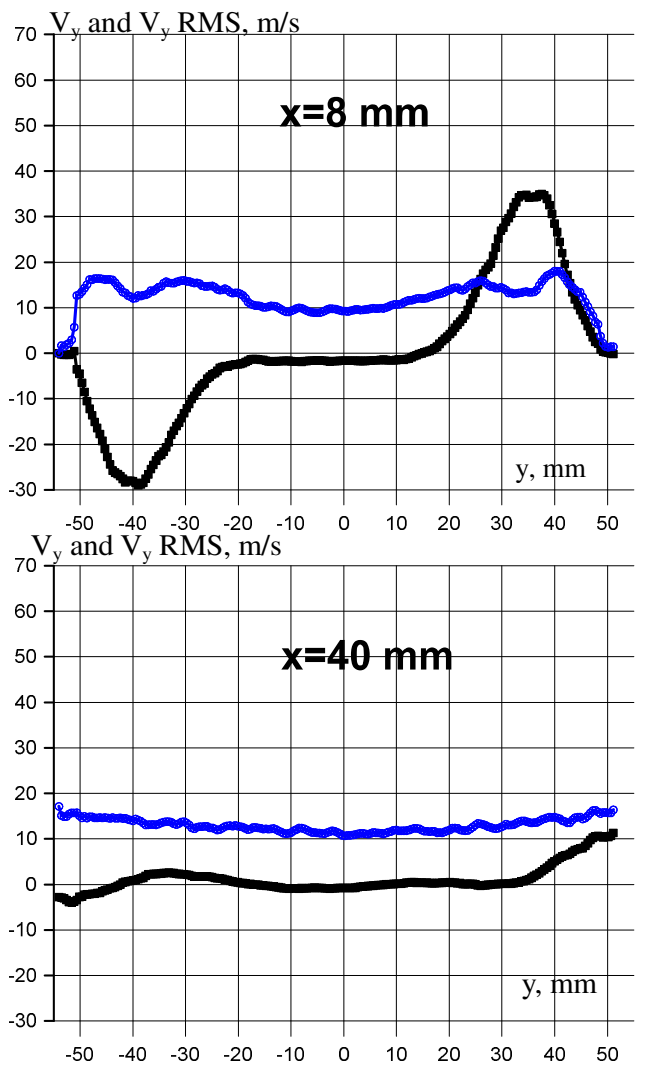

Figure 9. Radial profiles of the mean (black) and RMS (blue) values of $V y$ velocity component
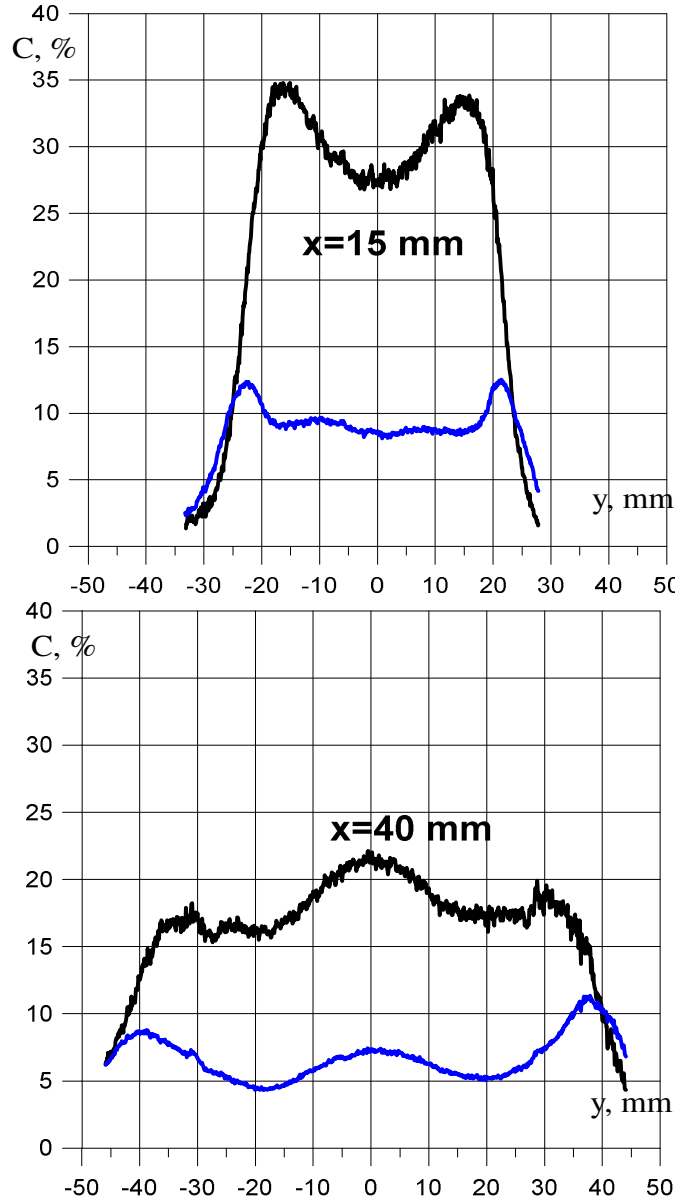

Figure 10. Concentration profiles (black line) and concentration RMS (blue line) at different distances from the burner exit

\subsection{Turbulent kinetic energy dissipation rate}

A turbulent kinetic energy (TKE) dissipation rate plays an important role in theories of turbulence and if recovered from experimental data can improve an overall understanding of the turbulent flow features.

TKE dissipation rate per unit volume depends on local kinematic viscosity of the fluid and strain rate tensor $S_{i j}$ :

$$
S_{i j}=\frac{1}{2}\left(\frac{\partial u_{i}}{\partial x_{j}}+\frac{\partial u_{j}}{\partial x_{i}}\right)
$$

And TKE dissipation rate is:

$$
\varepsilon=2 v\left\langle S_{i j} S_{i j}\right\rangle
$$

Expressed in simple algebraic form, equation (7) will contain 12 second order-moments of velocity derivatives. Thus, in order to obtain TKE dissipation rate directly from the experiment, all 3 components of velocity vector should be measured, and measurement spatial resolution should be sufficient to resolve finest spatial scales in the flow without introducing truncation error in spatial derivatives calculation. Usually such experimental data is unavailable, especially for high-Reynolds number flows: velocity data may be incomplete, contain noise and lack spatial resolution. In some cases, proper physical 
assumptions can be applied to reduce number of velocity components needed to estimate TKE dissipation rate.

As PIV data in the experiments under consideration contained only two velocity components in XY plane, direct TKE dissipation rate calculation from eq.7 wasn't possible. At the same time, according to the statistical data yielded from measurements taken in $\mathrm{YZ}$ plane, an assumption of rotational symmetry about $\mathrm{X}$ axis was quite appropriate despite of some skewness. Under this assumption formulation proposed by Hussain et. al. in [7] for TKE dissipation rate calculation can be applied. In this formulation only four second order-moments of velocity derivatives are used:

$\varepsilon=v\left[-\left\langle\left(\frac{\partial u_{1}}{\partial x_{1}}\right)^{2}\right\rangle+2\left\langle\left(\frac{\partial u_{1}}{\partial x_{2}}\right)^{2}\right\rangle+2\left\langle\left(\frac{\partial u_{2}}{\partial x_{1}}\right)^{2}\right\rangle+8\left\langle\left(\frac{\partial u_{2}}{\partial x_{2}}\right)^{2}\right\rangle\right]$

Eventually, if velocity derivatives calculated from experimental data using least-square scheme is substituted in eq. (8), $\varepsilon$ yielded from this equation, here and below addressed as $\varepsilon^{m}$, will qualitatively represent distribution of TKE dissipation rate, but quantitatively will greatly underestimate the value. The latter statement can be indirectly confirmed by the following considerations: coarse estimation of Kolmogorov's scale $\eta$ as $R e^{-3 /} 4$ yields $\eta \approx 50 \mu m$, while PIV spatial resolution, taking into account interrogation window overlap, was $\approx$ $1.8 \mathrm{~mm}$, besides, additional spatial filtering was introduced by numerical differentiation scheme. Consequently, for such measurement data large part of the spectrum falls into sub-grid scale, and, therefore, missed in TKE dissipation rate estimation.

To assess an «underestimation factor» a LES-based approach [8] together with appropriate model of turbulence can be applied. In this approach turbulence is assumed to conform to the selected model and limited spatial resolution of the measurement technique considered as a low-pass filter. Though PIV spatial filter is, in general, behaves like a cardinal sine function [9], for simplicity here it will be considered a sharp cut-off filter with $k_{\max }=\pi / \Delta$, where spatial resolution is homogeneous in $x$ and $y$ directions $\Delta=\Delta_{x}=\Delta_{y}$. For the case, $\Delta$ was equal to $8 h$, where $h$ is a step between velocity vectors in a PIV velocity field. Factor 8 for the $\Delta$ was chosen because of $75 \%$ window overlap in PIV interrogation and decrease in effective spatial resolution for velocity derivatives due to selected numerical differentiation scheme.

The Smagorinsky model for turbulent viscosity $v_{\tau}$ is:

$$
v_{\tau}=C_{S}^{2} \Delta^{2} \sqrt{\left(2 \overline{S_{i j}^{*}} \overline{S_{i j}^{*}}\right)}
$$

Where $S_{i j}$ is strain rate tensor, $\Delta$ is the filter width, $C_{S}$ is the Smagorinsky coefficient, and symbol $*$ indicates instantaneous quantity. According to [10] and some other works ensemble averaging operator can be introduced on the norm of the strain-rate tensor without loss of the models performance:

$$
v_{\tau} \approx C_{S}^{2} \Delta^{2}\left\langle 2 \overline{S_{i j}^{*}} \overline{S_{i j}^{*}}\right\rangle^{1 / 2} \approx C_{S}^{2} \Delta^{2}\left\langle 2 \overline{S_{i j} S_{i j}}\right\rangle^{1 / 2}
$$

Under the assumption of infinite $R e$ TKE dissipation rate can be expressed from eq. 7 as

$$
\varepsilon \approx 2 v_{\tau}\left\langle\overline{S_{i j}^{*}} \overline{S_{i j}^{*}}\right\rangle \approx 2 v_{\tau}\left\langle\overline{S_{i j}} \overline{S_{i j}}\right\rangle
$$

For the inertial range spectrum, filtered by a sharp cut-off filter with width $=\Delta$ the value of $C_{S}$ can be obtained as:

$$
C_{S}=\frac{1}{\pi}\left(\frac{2}{3 C}\right)^{3 / 4} \approx 0.173
$$

where $C \approx 1.5$ is Kolmogorov constant. In [11] other approximations, including an approximation for the case $L / \Delta>1$ (13) were proposed:

$$
\begin{gathered}
\varepsilon=2 v_{\tau}\left\langle\overline{S_{i j}^{*} \overline{S_{i j}^{*}}}\right\rangle, \\
v_{\tau}=\sqrt{\left(C_{s, \infty} \Delta / \gamma\right)^{4}\left(2 \overline{S_{i j}^{*}} \overline{S_{i j}^{*}}\right)+v^{2}}-v
\end{gathered}
$$

In this case, turbulent kinetic energy dissipation is given as a sum of sub-grid-scale and resolved dissipation, and $\gamma$ depends on the filter shape and is equal to a unit for a sharp cutoff filter.

Recalling that resolved scales in this analysis corresponds to that measured with PIV, a relation (15) with classic Smagorinsky or modified model for turbulent viscosity can be applied to correct data for the amount of TKE dissipation rate at not resolved scales:

$$
\begin{gathered}
\varepsilon=v_{\tau} \varepsilon^{m} / v, \text { where } \\
v_{\tau}=C_{S}^{2} \Delta^{2}\left(\varepsilon^{m} / v\right)^{1 / 2} \\
\text { or } \\
v_{\tau}=\sqrt{\left(C_{s} \Delta / \gamma\right)^{4}\left(\varepsilon^{m} / v\right)+v^{2}}-v
\end{gathered}
$$

In accordance with the model described above a «correction factor» of the TKE dissipation rate over the measurement area was calculated and «corrected» TKE dissipation rate was assessed (fig. 11). Predictably, an area of the maximum TKE dissipation rate corresponds to the region of most intense large-scale eddy interaction with the small-scale turbulence in the mixing layer. A «correction factor» exceeds 600 over the whole measurement area and in the mixing region goes as high as $10^{3}$. Eventually such results, in particular - extremely high «correction factor» mean that that only general features of the flow were fetched, and TKE dissipation rate assessment is valid to the order of magnitude at best. Generally it is not surprising as $R e$ was high $\left(\approx 3 \cdot 10^{5}\right)$ and PIV spatial resolution was quite low. 

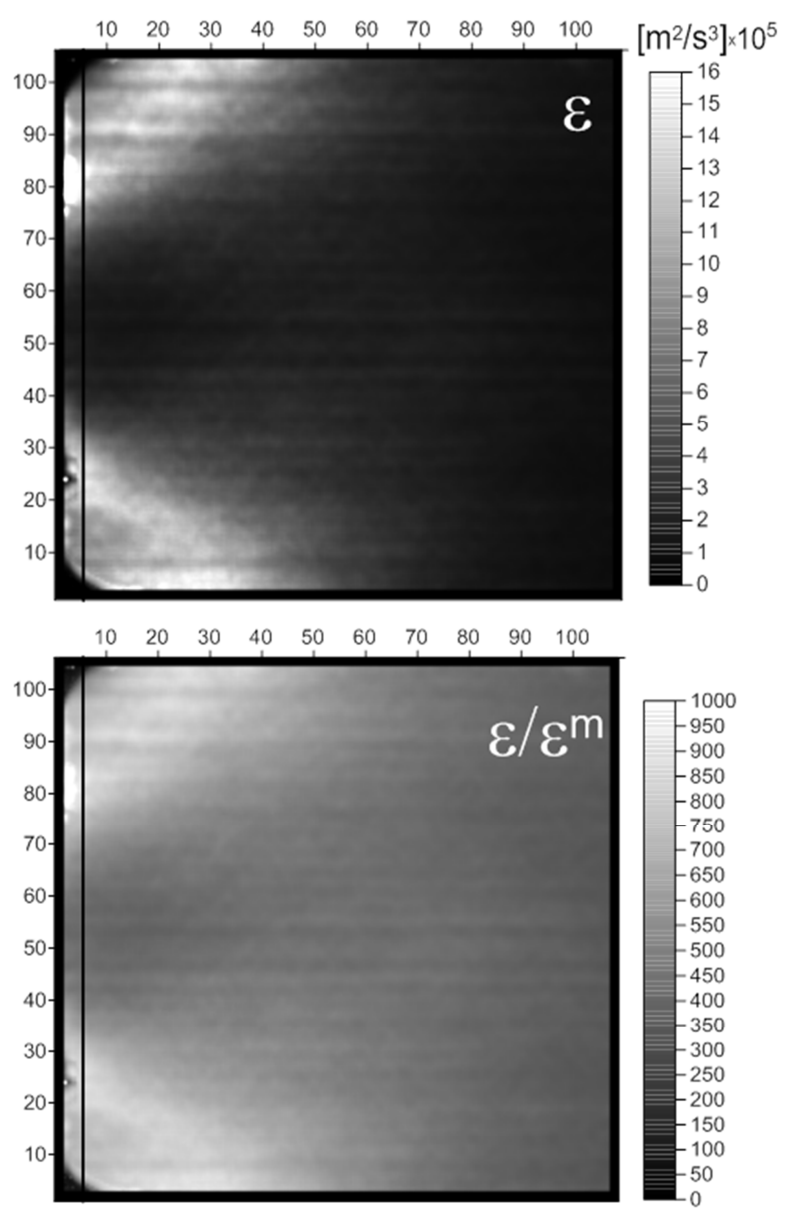

Figure 11. Distribution of TKE dissipation rate and «correction factor» over the measurement area in XY plane

\subsection{Turbulent mixing characteristics}

A conservation equation of species for turbulent flows can be written as:

$$
\frac{\partial \bar{c}}{\partial t}+\bar{u}_{j} \frac{\partial \bar{c}}{\partial x_{j}}=\frac{D \partial^{2} \bar{c}}{\partial x_{j}^{2}}-\frac{\partial\left\langle u_{j} c\right\rangle}{\partial x_{j}}
$$

where $\bar{c}$ is the time-averaged concentration, $c$ - the concentration fluctuation, $\bar{u}_{j}$ - time averaged velocity component, $u_{j^{-}}$velocity fluctuation component, $D$ molecular diffusion coefficient.

Right-hand part of the equation represents mass transport. In high-Reynolds number turbulent flows molecular diffusion term is usually negligible comparing to the turbulent diffusion term $\left\langle u_{j} c\right\rangle$ and can be omitted [12]. Than conservation equation takes following form:

$$
\frac{\partial \bar{c}}{\partial t}+\bar{u}_{j} \frac{\partial \bar{c}}{\partial x_{j}}=-\frac{\partial\left\langle u_{j} c\right\rangle}{\partial x_{j}}
$$

For this equation in CFD models a closure problem is usually solved by the assumption that turbulent mass transport is proportional to the mean concentration gradient - so called Fick's diffusion law assumption:

$$
\left\langle u_{j} c\right\rangle=-D t_{j} \frac{\partial \bar{c}}{\partial x_{j}}
$$

Yet, to validate the model for certain type of the flow fidelity of this assumption should be performed. For the experiments under consideration validation of Fick's law assumption was performed using PIV and PLIF experimental data.

In fig. 12 a distribution of y-component of $\left\langle u_{j} c\right\rangle$ and $y$-component of the mean admixture concentration gradient are presented. An area of the reversed flow is bounded by the solid line in the figure. Using these two distributions a turbulent admixture diffusion coefficient Dt can be assessed.
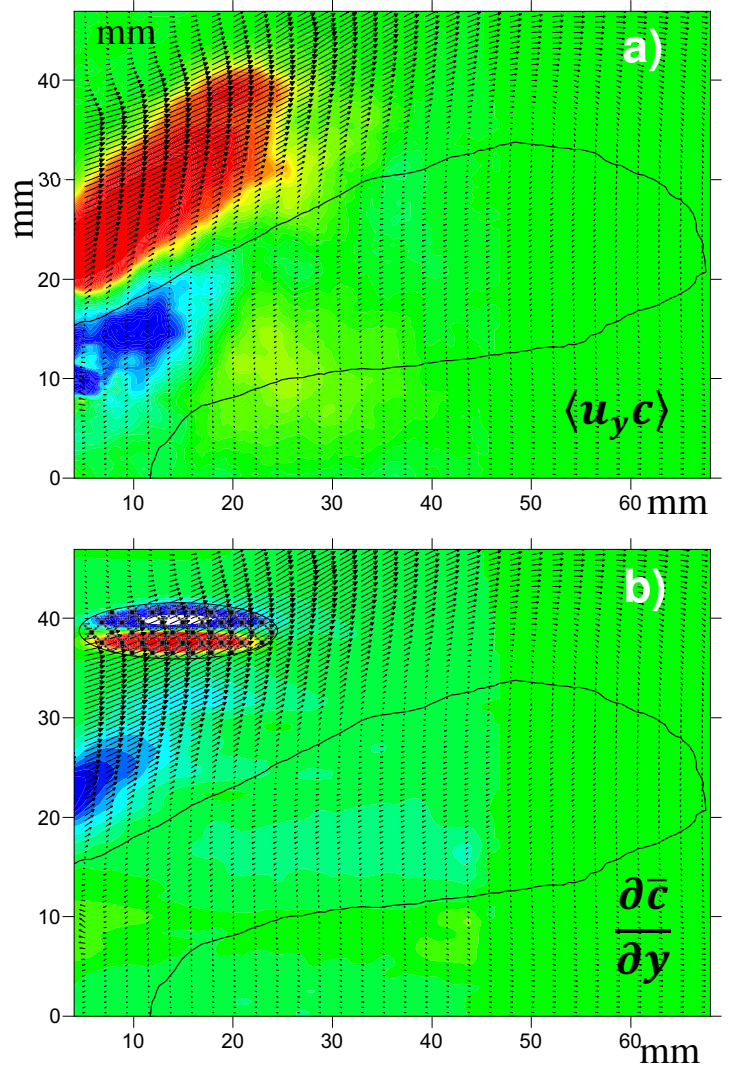

Figure 12. y-component of $\left\langle u_{j} c\right\rangle$ and y-component of the mean admixture concentration gradient

In fig.13 a profile of $y$-component of $\left\langle\mathrm{u}_{\mathrm{j}} \mathrm{c}\right\rangle$ and $y$ component of the mean admixture concentration gradient across the measurement area is shown. It can be seen from the figure that directly measured turbulent transport is in good correspondence with the Fick's diffusion law. Turbulent diffusion coefficient $D t$, that can be evaluated from such data by simple fitting of mean admixture concentration gradient profile to the $\left\langle\mathrm{u}_{\mathrm{j}} \mathrm{c}\right\rangle$, was equal to 0.028 for the case. 


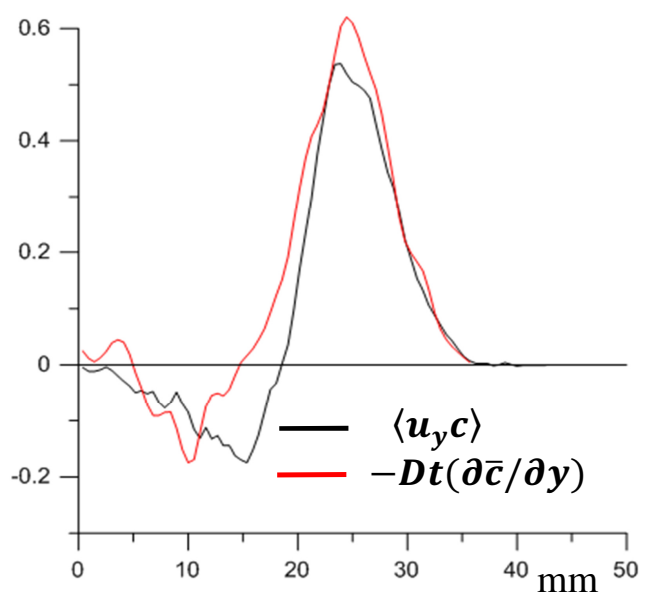

Figure 13. Profile of y-component of $\left\langle u_{j} c\right\rangle$ and $y$-component of the mean admixture concentration gradient across the measurement area.

Another representative characteristic that can be recovered from simultaneous PIV/PLIF measurements is turbulent Schmidt number, which represents the ratio between the rates of turbulent transport of momentum and the turbulent mass transport.

As Schmidt number $S c_{t}=v_{t} / D t$, a distribution of $v_{t}$ is required to calculate $S c_{t}$ over the measurement area. Such distribution is available from PIV data through distribution of Reynolds stress and mean velocity gradient component, which are presented in fig. 14. As can be seen from the figure, maximum and minimum of both differ in sign and are reached within the shear layers of the swirling flow.
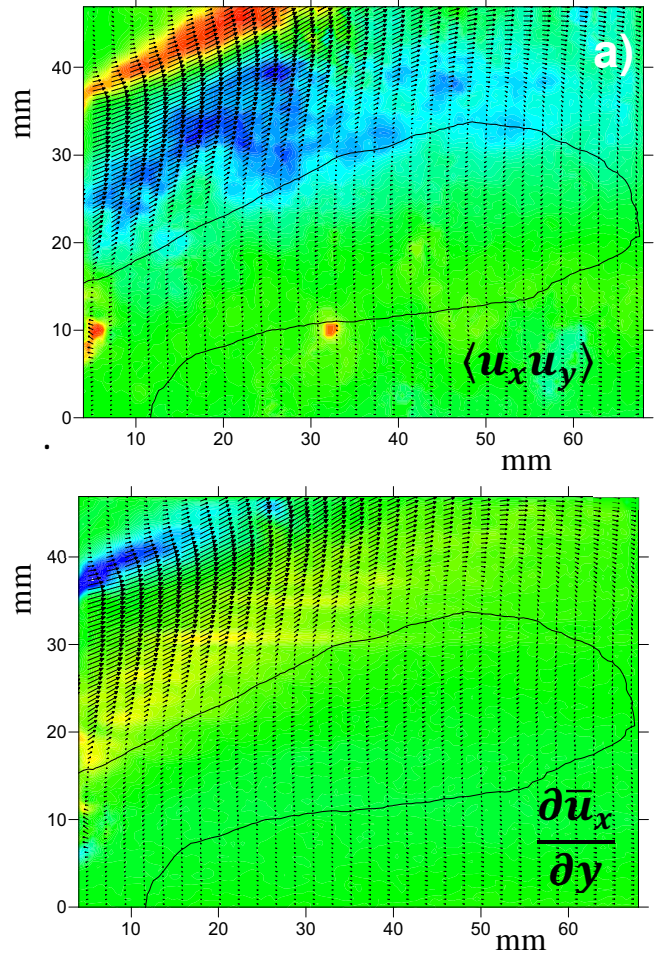

Figure 14 distribution of Reynolds stress component (a) and mean velocity gradient component (b)
Again, like in the previous case, turbulent viscosity $v_{t}$ can be evaluated from experimental data by simple fitting of mean velocity gradient profile to the profile of Reynolds stresses (see fig. 15). Such evaluation yields $v_{t} \approx 0.01$, and corresponding $S c_{t}$ value of 0.35 .

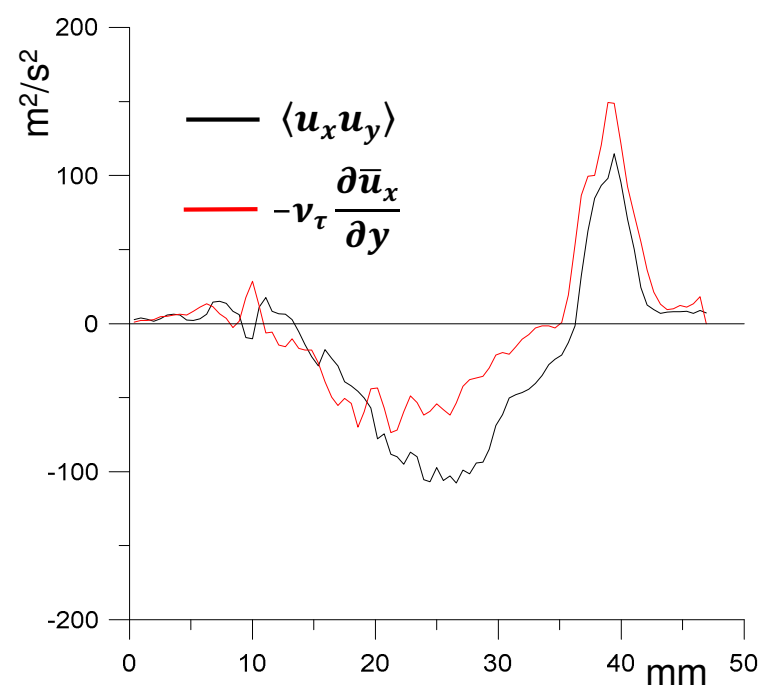

Figure 15. Profile of Reynolds stress component of $\left\langle u_{x} u_{y}\right\rangle$ and $y$-component of the mean streamwise velocity component gradient

\section{Conclusion}

In the present work combined PLIF/PIV experiments were carried out in a cold model of GT-burner at realistic flow-rates. The results include instantaneous simultaneously measured 2D concentration of passive admixture and velocity fields. The main goal of the investigation was a better understanding of the turbulent mixing process in an industrial burner and acquisition of experimental data for validation of numerical simulation. Despite of non-perfect quality of quartz glass cylinder and multiple laser sheet reflections data processing algorithms allowed to get reliable experimental data. The results indicate that there is a strong correlation between velocity and concentration fields.

In addition evaluation techniques for assessment of TKE dissipation rate and turbulent passive admixture transport for the case were considered. TKE dissipation rate was assessed from 2D-PIV data using an assumption of rotational symmetry, though assessment precision was rather low. Turbulent diffusion coefficient and turbulent viscosity, required for application of gradient closure hypothesis and evaluation of turbulent Schmidt number were retrieved from the PIV/PLIF simultaneous measurements. It was also shown, that in general, Reynolds stresses and turbulent passive admixture flux, both conform to the gradient closure hypothesis. 


\section{Acknowledgments}

This work was funded by a grant from the Russian Science Foundation (project No. 14-29-00203 in NSU, supervised by Professor Kemal Hanjalic).

\section{References}

1. A. Lozano, S.H. Smith, M.G. Mungal, and R.K. Hanson, AIAA J., 32, 218 (1993)

2. L.K Su., M.G. Mungal, 43rd Aerospace Science Meeting and Exhibit, AIAA 2005-305, (2005)

3. M. Raffel, C. Willert, S. Wereley, J. Kompenhans, Particle Image Velocimetry. A practical guide, Second Edition (2007)

4. S. Alekseenko, A. Bilsky, V. Dulin, B. Ilyushin, D. Markovich, Non-intrusive determination of turbulent energy balance in free and confined jet flows. 4th International Symposium on Turbulence and Shear Flow Phenomena, Williamsburg, VA, USA (2005)

5. J.M. Foucaut, B. Milliat, N. Perenne, M. Stanislas, Characterization of different PIV algorithms using the EUROPIV Synthetic Image Generator and real images from a turbulent boundary layer EUROPIV 2 Workshop on Particle Image Velocimetry, 163 (2004)

6. R.A. Bryant, J.M. Donbar, J.F. Driscoll, Exp. Fluids 28, №. 5, 471(2000).

7. W.K. George, H.J. Hussein, 233, 1 (1991)

8. V.M. Dulin, D.M. Markovich, S.V. Alekseenko, Stereo PIV measurements of fine-scale turbulence statistics in a free jet flow Proc. 6th Int. Symp. Turbulence, Heat Mass Transfer, Begell House, Rome (2009).

9. J.M. Foucaut, J. Carlier, M. Stanislas, Meas Sci Technol 15, 1046, (2004)

10. O. J. McMillan, J. H. Ferziger, AIAA J. 17, 1340 (1979).

11. J. Meyers, P. Sagaut, J. Fluid Mech. 569, 287, (2006)

12. P. J. W. Roberts, D. R. Webster, Turbulent diffusion, In: Environmental Fluid Mechanics Theories and Application, 7 (2002) 